\title{
Parkinsonism in Lacunar Infarcts of the Basal Ganglia
}

\begin{tabular}{|l|l|l|}
\hline K. & Ken & Ikeda $^{\mathrm{a}}$ \\
\hline T. & Tetsuo & Kotabe $^{\mathrm{b}}$ \\
\hline S. & Sho & Kanbashi $^{\mathrm{b}}$ \\
\hline M. & Masao & Kinoshita $^{\mathrm{a}}$ \\
\hline
\end{tabular}

${ }^{\mathrm{a}} 4$ th Department of Internal Medicine and ${ }^{\mathrm{b}}$ Division of Rehabilitation, Toho University Ohashi Hospital, Tokyo, Japan Ken Ikeda, MD, PhD, 4th Department of Internal Medicine, Toho University Ohashi Hospital, 2-17-6 Ohashi, Meguro-ku, Tokyo 153 (Japan)

We read with interest the article by Reider-Groswasser et al. [1], concerning 'parkinsonism in patients with lacunar infarcts of the basal ganglia', and we agree with most of their conclusions. We have also encountered several patients with parkinsonism due to lacunar infarcts. We would like to compare our findings with their results [1] and then pose several questions.

Patient 1

A 76-year-old hypertensive woman developed gait disturbance in 1992. Her gait disturbance gradually progressed, and she came to our department in July 7, 1994. General physical examination was normal except for hypertension $(170 / 90 \mathrm{~mm} \mathrm{Hg})$. Neurological examination showed slow and small-stepped gait with initiating hesitation. Voluntary movements in the upper limbs and fingers were excellent. Involuntary movements were absent. Muscle stretch reflexes were normal without Babinski signs. The muscle tonus was normal in the extremities. The rest of her neurological examination, including cognitive, speech, cerebellar, and bladder functions, was normal. Computed tomography (CT) revealed multiple lacunar infarcts in the basal ganglia. Magnetic resonance imaging (MRI) showed multiple lacunar infarcts in basa ganglia, thalamus, internal capsules, and periventricular regions (fig. 1A). We diagnosed parkinsonism in the lower body, due to multiple lacunar infarcts. Oral levodopa/carbidopa (300 mg/day) was not effective to treat her parkinsonism. When she received amantadine $150 \mathrm{mg} /$ day p.o. her gait disturbance was moderately retarded.

Patient 2

A 70-year-old man with hypertension had a history of small-stepped gait of 2 years. On neurological examination, his gait was very slow and small stepping with initiating hesitation. Slight bradykine-sia, but not tremor presented in the upper extremities. The muscle tonus was normal in the extremities. The rest of his neurological examination, such as cognitive function, speech, pyramidal tract signs, sensory function, and cerebellar ataxia, was normal. CT showed lacunar infarcts in the basal ganglia. A $\mathrm{T}_{2}$-weighted image disclosed high signal intensity areas in putamen, caudate nuclei, thalamus, internal capsules, and periventricular areas. Another $\mathrm{T}_{2}$-weighted image showed hypointensity signal areas in right putamen and left thalamus (fig. IB). These MRI findings indicated lacunar infarcts and small hemorrhages. Brain HMPAO single-photon emis-
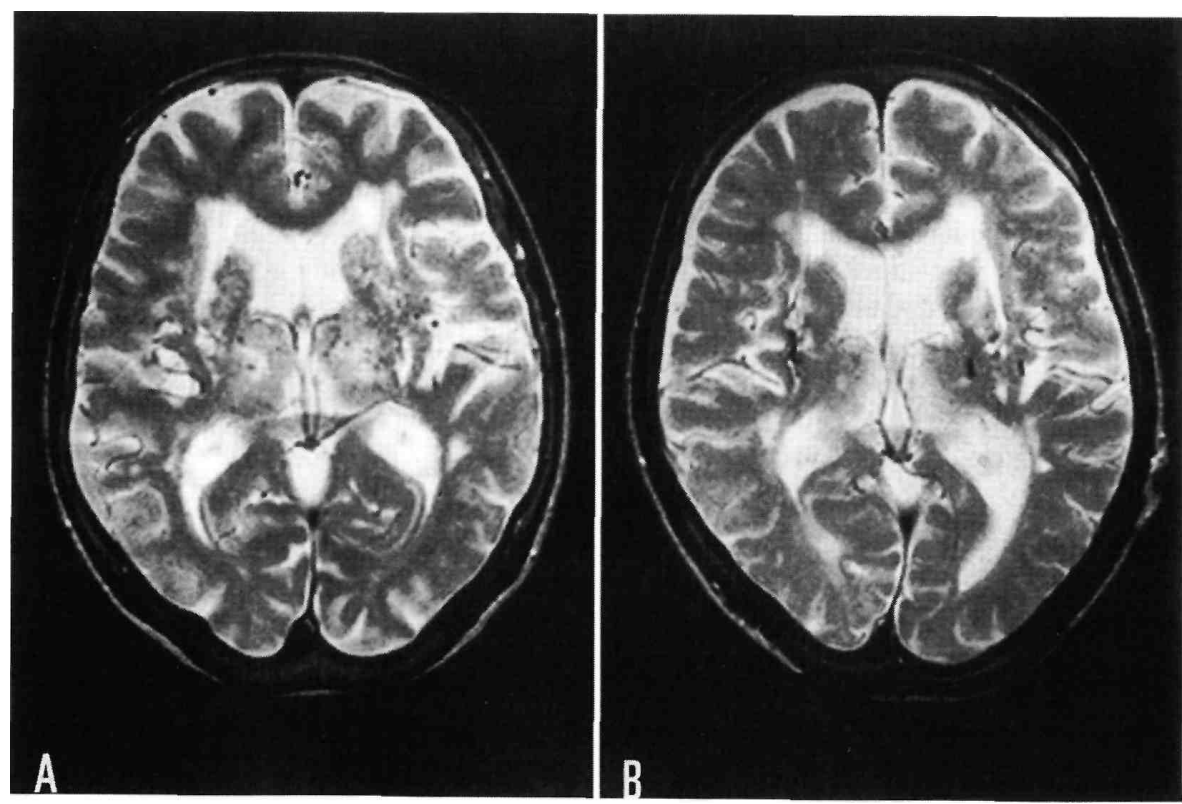
Fig. 1. Axial $\mathrm{T}_{2}$-weighted images. The left side of the image corresponds to the patient's right side. A Patient 1 . $\mathrm{T}_{2}$-weighted image showing lacunae in basal ganglia, thalamus, internal capsules, and periventricular regions. B Patient 2. $\mathrm{T}_{2}$-weighted image high signal intensity areas in putamen, caudate nuclei, thalamus, internal capsules, and periventricular areas. Hypointensity signal areas are also detected in right putamen and left thalamus.

248 
sion CT (SPECT) showed diffuse cortical hypoperfusion. Levodopa/ carbidopa (300 mg/day p.o.) treatment was not beneficial to his gait disturbance. A diagnosis of lower body predominant vascular parkinsonism was made. His gait recovered mildly under amantadine $(150 \mathrm{mg} /$ day $)$ therapy.

The clinical features of both patients were latent onset and lower body predominant parkinsonism. Furthermore, on neuroimaging, CT showed only multiple lacunar infarcts limited in the basal ganglia. However,

MRI indicated lacunae in thalamus, internal capsule, and periventricular regions, in addition to the basal ganglia. Small hemorrhage was also associated in our patient 2.

Reider-Groswasser et al. [1] suggested the possibility that the damage to the internal capsule causes the high prevalence of hemi-paresis in their patients with parkinsonism. CT was not sufficient to exclude such lesions.

We would like to know the findings of MRI and SPECT in their patients, if they performed such studies. CT in our patients failed to detect small lacunae or hemorrhages in other than the basal ganglia. 
SPECT showed decreased cerebral blood flow of the cerebral cortex in our patient 2, whose CT showed lacunae confined to the basal ganglia. The second question is whether there is any predominance between upper and lower limbs in patients with parkinsonism, although Reider-Groswasser et al. [1] and Inzelberg et al. [2] reported the clinical features of disturbance. We speculate that lower body predominant disability is a symptomatic feature in patients with parkinsonism due to multiple lacunar infarcts.

\section{References}

Reider-Groswasser I, Bornstein NM, Korczyn AD: Parkinsonism in patients with lacunar infarcts of the basal ganglia. Eur Neurol 1995·35:46-49.

2 Inzelberg R, Bornstein NM, Reider I, Korczyn AD: Basal ganglia lacunes and parkinsonism. Neuroepidemiology 1994;13:108-112. 
